\title{
Serum alpha 1-antitrypsin predicts severe acute kidney injury after cardiac surgery
}

\author{
Songlin Du ${ }^{1 \#}$, Jianwei Tian ${ }^{2 \#}$, Zhiwen Xiao ${ }^{2}$, Zhiwen Luo ${ }^{1}$, Tong Lin ${ }^{2}$, Shaoyi Zheng ${ }^{1}$, Jun Ai $^{2}$ \\ ${ }^{1}$ Department of Cardiovascular Surgery, Nanfang Hospital, Southern Medical University, Guangzhou 510515, China; ${ }^{2}$ State Key Laboratory of \\ Organ Failure Research, National Clinical Research Center of Kidney Disease, Division of Nephrology, Nanfang Hospital, Southern Medical \\ University, Guangzhou 510515, China \\ Contributions: (I) Conception and design: S Du, J Ai; (II) Administrative support: J Ai; (III) Provision of study materials or patients: S Du, S Zheng; (IV) \\ Collection and assembly of data: J Tian, Z Xiao, Z Luo, T Lin; (V) Data analysis and interpretation: S Du, J Ai; (VI) Manuscript writing: All authors; \\ (VII) Final approval of manuscript: All authors. \\ "These authors contributed equally to this work. \\ Correspondence to: Jun Ai, MD, PhD. Division of Nephrology, Nanfang Hospital, Southern Medical University, No. 1838 North Guangzhou Ave, \\ Baiyun District, Guangzhou 510515, China. Email: aij1980@163.com.
}

\begin{abstract}
Background: Human alpha 1-antitrypsin (A1AT) is involved in the pathophysiological process underlying ischemic acute kidney injury (AKI). To test the hypothesis that serum A1AT (sA1AT) is a predictor for severe AKI after cardiopulmonary bypass (CPB), we conducted a prospective cohort study in 201 patients undergoing cardiac surgery.

Methods: We collected blood and urine samples, and analyzed the sA1AT and other injury biomarkers during the perioperative period. Severe AKI is defined as Kidney Disease Improving Global Outcomes (KDIGO) stage 2 or 3, and overall AKI is defined as KDIGO stage 1, 2, or 3.

Results: Ninety-one (45.3\%) patients developed overall AKI, and 22 (10.9\%) among them developed severe AKI after operation. sA1AT level spiked 2 hours after surgery in patients who subsequently developed severe AKI, while serum creatinine peaked 12 hours after operation. Higher postoperative sA1AT independently correlated to the development of severe AKI [OR, 1.54 (1.17-2.03); P=0.002]. The highest quartile of postoperative sA1AT level was associated with 6-fold higher hazards of severe AKI compared to the lowest quartile. Higher sA1AT levels were correlated with longer stays in the intensive care unit and the hospital. For predicting severe AKI, the AUC of sA1AT 2 hours after CPB reached 0.814. After combining with urine T cell immunoglobulin mucin-1 and clinical model, the AUC improved to 0.923 .
\end{abstract}

Conclusions: In summary, sA1AT is a valuable predictor of severe AKI development and prolonged ICU and hospital stays in patients after cardiac surgery.

Keywords: Serum alpha 1-antitrypsin (sA1AT); predictor; acute kidney injury (AKI); cardiac surgery; cardiopulmonary bypass (CPB)

Submitted Sep 10, 2019. Accepted for publication Nov 12, 2019.

doi: $10.21037 /$ jtd.2019.12.17

View this article at: http://dx.doi.org/10.21037/jtd.2019.12.17

\section{Introduction}

Acute kidney injury (AKI) is a common complication which is reported frequently among $30 \%$ of post-cardiac surgeries in adults $(1,2)$, and is associated with higher risk of mortality and poor long-term adverse events (3-7). Even though severe kidney injury is reported in many cases before the elevation of serum creatinine levels, clinicians mainly rely on serum creatinine levels as the functional biomarker for assessing clinical diagnosis and staging of AKI (8). Earlier detection of severe AKI would afford clinicians an earlier 
time window to intervene proper therapeutic management in patients undergoing cardiac surgery.

The human alpha 1-antitrypsin (A1AT) is an acute phase glycoprotein with broad immunoregulatory and antiinflammatory property, which showed a higher binding efficiency with hemin that prevents the formation of hemininduced reactive oxygen species (ROS) in neutrophils (9-12). It was previously reported that A1AT is involved in the pathophysiologic processes underlying ischemic AKI, and this redox-sensitive stress protein can exert dramatic renoprotective effects (13). Recently, an abundance of experimental data supported the concept that serum A1AT (sA1AT) was a potential marker of neutrophil activation, and the signal pathway was activated in patients with AKI induced by ischemia-reperfusion injury or renal toxicity (12-14). We therefore hypothesized that sA1AT might be associated with severe AKI and act as a noninvasive biomarker for early prediction of AKI after cardiopulmonary bypass (CPB).

In order to test and confirm the hypothesis, we have conducted a prospective cohort study involving 201 patients undergoing $\mathrm{CPB}$ and evaluated the performance of sA1AT compared with other selected biomarkers for predicting severe AKI in patients after cardiac surgery.

\section{Methods}

\section{Patients}

This was a prospective, single-center, cohort study approved by the Department of Cardiovascular Surgery, Nanfang Hospital, Southern Medical University. All the participants enrolled in this study provided written informed consent (ethics number NFEC-201509-K2, approved by the Research Ethics Committee of Nanfang Hospital). The study was conducted from 1 July to 31 December, 2017. All patients undergoing elective cardiac surgery with $\mathrm{CPB}$ between 18-80 years of age were eligible for this study. The exclusion criteria included exposure to pre-existing advanced CKD [chronic dialysis, renal transplantation, or pre-operative estimated glomerular filtration rate (eGFR) $<30 \mathrm{~mL} / \mathrm{min}$ per $1.73 \mathrm{~m}^{2}$ ], exposure to nephrotoxin drug therapies (receiving contrast media caused AKI, aminoglycoside antibiotics, vancomycin, and non-steroidal antiinflammatory drugs [except aspirin]) within 4 weeks prior to surgery, and urinary tract infection or obstruction.

We collected spot urine and blood samples before operation (as baseline spot) and 2, 12, 24, and 48 hours after CPB. When CPB time exceeded 2 hours, sample was collected at the end of operation and was regarded as the first point after operation sample. All samples were centrifuged at 3,000 g for 10 minutes. The supernatant liquids were stored at $-80{ }^{\circ} \mathrm{C}$ after labeled using study identification numbers without personal identifiers or clinical conditions. Serum creatinine was measured to determined changes in renal function. Clinical data were collected at the same time.

\section{Measurement of biomarkers}

All biomarkers were measured in our central key laboratory following the standard protocol. Serum and urine A1AT levels and urine $\mathrm{T}$ cell immunoglobulin mucin-1 (uTIM-1) were measured at baseline, and 2, 12, 24, 48 hours after operation by ELISA kits (ab189579; Abcam, Cambridge, UK; and DY1750B; R\&D Systems, Minneapolis, USA) according to manufacturer's instructions. All biomarkers were measured in triplicate. The intra- and inter- assay variability ranged between $3-6 \%$ and $2-8 \%$ on the basis of blinded replicate samples from study patients, respectively.

Serum and urine creatinine were measured using an automatic biochemical analyzer (AU480; Olympus, Tokyo, Japan). All of the urinary biomarkers were normalized by urine creatinine.

\section{Outcome definitions}

The primary outcome was the development of severe AKI, which was defined as a 2 -fold increase in serum creatinine after surgery compared to baseline, or as receiving acute dialysis [Kidney Disease Improving Global Outcomes (KDIGO) stage 2 or 3] (15). Mild AKI was defined as a serum creatinine level increasing up to $26.5 \mathrm{mmol} / \mathrm{L}$ $(0.3 \mathrm{mg} / \mathrm{dL})$, but not a 2 -fold increase within 7 days (KDIGO stage 1) (15). Overall AKI was defined as severe AKI and mild AKI (KDIGO stage 1, 2, or 3) (15). The secondary outcome was the length of stay in the intensive care unit (ICU) and the hospital (including ICU stays).

\section{Statistical analyses}

Stata 15 software was used for the statistical analyses. The ANOVA or the Kruskal-Wallis test was used to compare continuous variables, and the chi-square test was used to compare categorical variables. For an overall comparison 


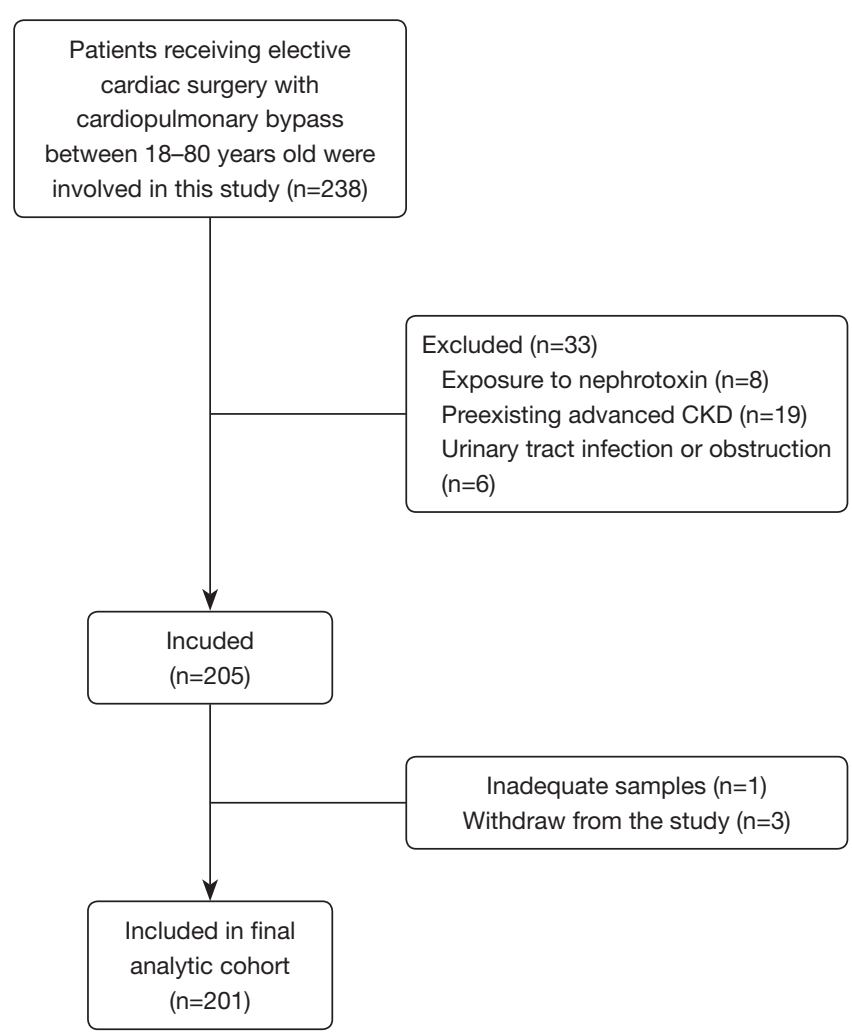

Figure 1 Flow diagram of patient selection into the study cohort.

of change over time between groups, a random effects model analysis was performed with particular focus on the interaction between group and time.

Logistic regression analyses were performed to determine the relationships between severe AKI and sA1AT or urine A1AT (uA1AT) 2 hours after CPB, and clinical variables. We categorized the sA1AT 2 hours after CPB into four groups, and then performed Kaplan-Meier and cox regression analyses on created variables. To compare the performance of biomarkers, an area under the receiver operating characteristic curve (AUC) was generated. Adjusted (age, sex, BMI, SBP, preoperative eGFR, and CPB time) linear regression was performed to analyze the relationship between sA1AT and length of stay in the ICU and the hospital. The Hosmer-Lemeshow (HL) goodness of fit and variance inflation factor (VIF) analyses were performed to evaluate the model performance and assessment of collinearity.

The continuous data are shown as the mean \pm standard difference (SD) or median [interquartile range (IQR)], the categorical data were shown as number (proportion), the odds ratio (OR) data were shown as OR $[95 \%$ confidence interval $(95 \% \mathrm{CI})]$, the hazard ratio (HR) data were shown as $\mathrm{HR}(95 \% \mathrm{CI})$, and the AUC data were shown as AUC (95\% CI). A $\mathrm{P}<0.05$ was considered to be statistically significant.

\section{Results}

\section{Cohort description}

Two hundreds and one patients subjected to cardiac surgery were included in the study followed by data analysis (Figure 1). All surgeries were elective and carried out in our center with CPB. Ninety-one (45.3\%) patients developed overall AKI, and 22 (10.9\%) among them developed severe AKI. Severe AKI occurred at 12 [2-12] hours after operation. Patients developing severe AKI were older, have a lower preoperative eGFR, lower contrast medium using, higher triple procedure, and longer $\mathrm{CPB}$ time than patients without AKI (Table 1).

\section{Serum A1AT as a predictor for developing severe AKI after $C P B$}

As shown in Figure 2, sA1AT spiked immediately 2 hours after operation, maintained at the peak for 12 hours, and then decreased, while the peak in serum creatinine were 12-24 hours after CPB. Even though post-operative sA1AT increased in all individuals, patients with severe AKI displayed a higher magnitude changes over time compared with those who had mild AKI $(\mathrm{P}=0.001)$ or had no AKI $(\mathrm{P}<0.001)$. Unlike sA1AT, the uA1AT did not change significantly over time in patients with or without AKI (Figure S1).

As shown in Table 2, the sA1AT 2 hours after operation [OR, $1.632(1.314-2.026)], \mathrm{P}<0.001)$ and $\mathrm{CPB}$ time [OR, $1.023(1.015-1.031), \mathrm{P}<0.001]$ significantly associated with incident of severe AKI. After adjustment for clinical model (age, gender, BMI, SBP, pre-operative eGFR) and $\mathrm{CPB}$ time, higher sA1AT independently correlated to the development of severe AKI [OR, 1.536 (1.165-2.025); $\mathrm{P}=0.002]$. Furthermore, as shown in Table S1, higher sA1AT independently associated with the development of overall AKI [OR, 1.231 (1.020-1.485); $\mathrm{P}=0.030]$.

We next categorized sA1AT 2 hours after operation into 4 groups (quartile). As shown in Table 3 and Figure 3, patients with the highest sA1AT quartile had the maximum incidence of severe AKI $(29.4 \%, \mathrm{P}<0.001)$ and overall AKI $(60.8 \%, \mathrm{P}<0.001)$. The highest quartile of the postoperative 
Table 1 Description of cohort after cardiac surgery

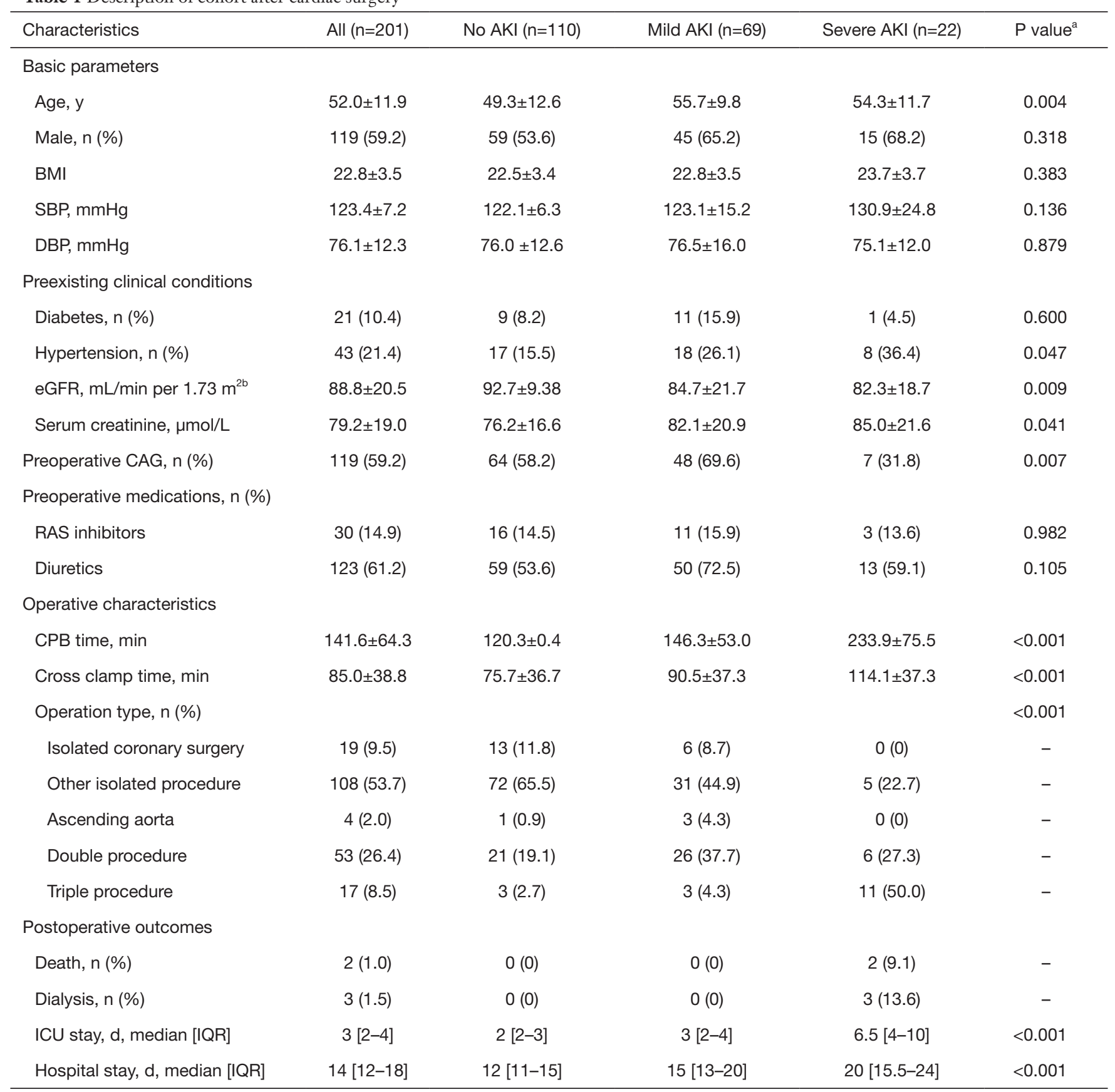

Continuous variables were expressed as mean \pm SD or median [IQR]. Categorical variables were expressed as $\mathrm{n}(\%) .{ }^{\mathrm{a}}, \mathrm{P}$ value for global comparisons among groups by Kruskal-Wallis and chi-squared tests for continuous and categorical variables, respectively; ', eGFR was determined by the Chronic Kidney Disease Epidemiology Collaboration Equation [2009]. AKI, acute kidney injury; BMI, body mass index; CAG, coronary angiography; CPB, cardiopulmonary bypass; eGFR, evaluated glomerular filtrated rate; ICU, intensive care unit; IQR, interquartile range; RAS, renin-angiotensin system; SBP, systolic blood pressure; SD, standard deviation. 
A

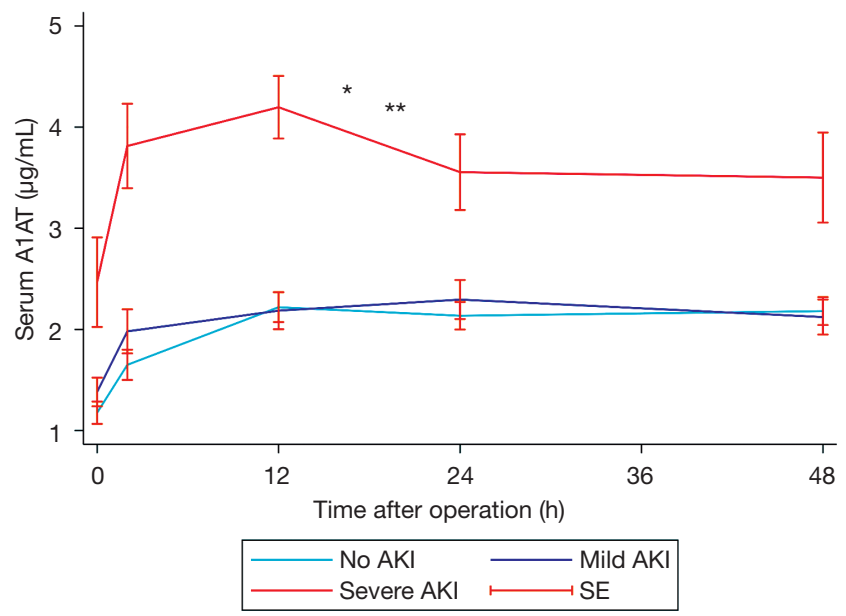

B

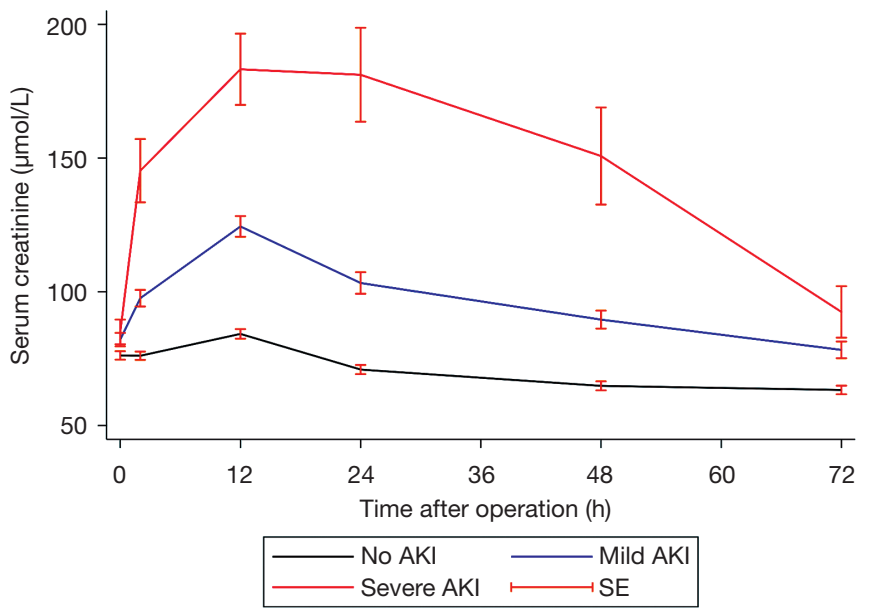

Figure 2 Changes of sA1AT (A) and serum creatinine (B) levels in patients over time after surgery. (A) Changes of sA1AT levels over time in patients with no AKI, mild AKI, and severe AKI; (B) changes of serum creatinine levels over time in patients with no AKI, mild AKI, and severe AKI. *, $\mathrm{P}=0.001$ versus mild AKI; ** $\mathrm{P}<0.001$ versus no $\mathrm{AKI}$. AKI, acute kidney injury; sA1AT, serum alpha1-antitrypsin.

Table 2 Multivariate logistic regression analyses of sA1AT for predicting severe AKI after cardiac surgery ${ }^{2}$

\begin{tabular}{|c|c|c|c|c|c|c|}
\hline Characteristics & \multicolumn{3}{|c|}{ Unadjusted } & \multicolumn{3}{|c|}{ Adjusted $^{b}$} \\
\hline sA1AT & 1.632 & $1.314-2.026$ & $<0.001$ & 1.536 & $1.165-2.025$ & 0.002 \\
\hline Age & 1.019 & 0.980-1.059 & 0.348 & 1.012 & $0.948-1.080$ & 0.703 \\
\hline Male & 0.671 & $0.251-1.664$ & 0.367 & 0.914 & $0.270-3.094$ & 0.886 \\
\hline SBP & 1.027 & $1.002-1.053$ & 0.033 & 1.012 & $0.980-1.045$ & 0.464 \\
\hline Baseline eGFR & 0.982 & $0.961-1.004$ & 0.116 & 0.995 & $0.962-1.028$ & 0.772 \\
\hline CPB time & 1.023 & $1.015-1.031$ & $<0.001$ & 1.022 & $1.012-1.031$ & $<0.001$ \\
\hline
\end{tabular}

a , sA1AT expressed 2 hours after CPB; ${ }^{\text {b }}$, adjusted for age, male, BMI, SBP, preoperative eGFR, and CPB time. AKI, acute kidney injury; $\mathrm{BMI}$, body mass index; $\mathrm{Cl}$, confidence interval; $\mathrm{CPB}$, cardiopulmonary bypass; eGFR, evaluated glomerular filtrated rate; OR, odds ratio; sA1AT, serum alpha1-antitrypsin; SBP, systolic blood pressure.

sA1AT levels was associated with increasing risk of severe AKI [HR, 16.77 (2.21-126.98), $\mathrm{P}=0.006]$ than the lowest quartile. Even adjusted by clinical variables, the highest quartile presented higher incidence of severe AKI than quartile 1, 2 and 3 (Tables 3,S2 and Figure 4A). Moreover, quartile of sA1AT levels showed a graded relationship with the incidence of overall AKI (Table $S 3$ and Figure 4B).

\section{Serum A1AT as a predictor for outcome}

We analyzed the lengths of ICU or hospital stay as secondary outcomes. The median length of stay was 3 [2-4] days in the ICU and 14 [12-18] days in hospital (Table 1). As shown in Table S4, higher sA1AT significantly associated with a longer stay in the ICU (P for trend 0.021) and in the hospital ( $\mathrm{P}$ for trend 0.002).

\section{Performance of $s A 1 A T$}

We next compared the performance of sA1AT with uTIM-1, a classic biomarker for early detection of AKI after cardiac surgery $(16,17)$. As shown in Table 4 and Figure 5, the AUC 
Table 3 Multivariate cox regression analyses of sA1AT for predicting severe AKI after cardiac surgery ${ }^{a}$

\begin{tabular}{|c|c|c|c|c|c|}
\hline sA1AT & $\begin{array}{l}\text { Severe } \\
\text { AKI (\%) }\end{array}$ & \multicolumn{4}{|c|}{ Severe AKI } \\
\hline 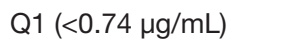 & 1.96 & 1.0 (reference) & - & 1.0 (reference) & - \\
\hline Q2 (0.74-1.21 $\mu \mathrm{g} / \mathrm{mL})$ & 2.0 & $1.02(0.06-16.31)$ & 0.989 & $0.80(0.05-13.07)$ & 0.875 \\
\hline Q3 (1.21-2.43 $\mu \mathrm{g} / \mathrm{mL})$ & 10.2 & $5.31(0.62-45.46)$ & 0.127 & $1.73(0.18-16.47)$ & 0.632 \\
\hline$P$ for trend & $<0.001$ & & & & \\
\hline
\end{tabular}
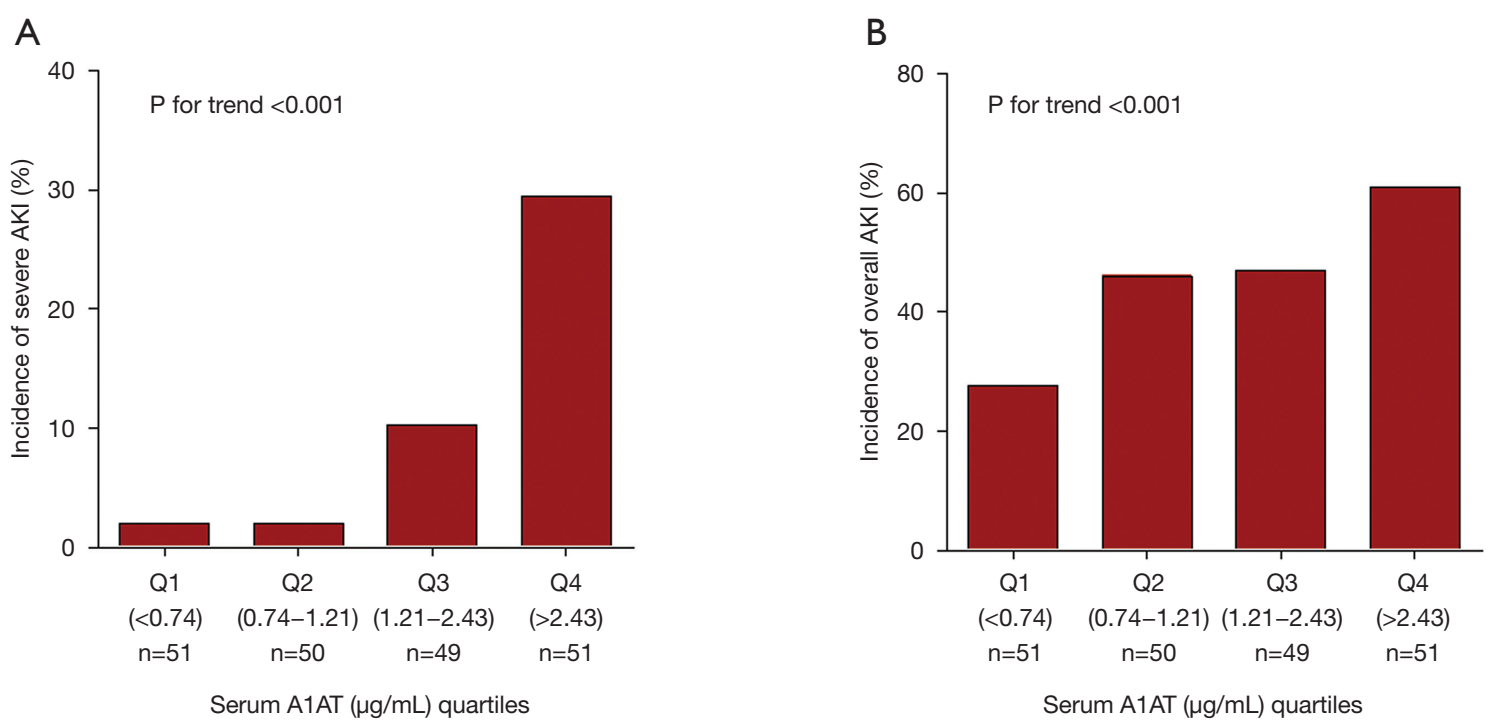

Figure 3 Quartile of sA1AT levels 2 hours after surgery had a graded relationship with incident severe AKI (A) and overall AKI (B). Patients with the highest quartile of the sA1AT levels had the highest incidence of severe AKI $(29.4 \%, \mathrm{P}<0.001$ for trend; A) and overall AKI (60.8\%, $\mathrm{P}<0.001$ for trend; B). AKI, acute kidney injury; $\mathrm{Q}$, quartile; sA1AT, serum alpha1-antitrypsin.

for predicting severe AKI of sA1AT was $0.814(0.732-0.896)$, which seemed better than uTIM1 [0.712 (0.591-0.833); $\mathrm{P}=0.136]$. For predicting overall AKI, the AUC of sA1AT was $0.628(0.550-0.705)$.

We further evaluated the performance of a combination of sA1AT 2 hours after operation with uTIM1 and clinical variables. The AUCs of sA1AT for predicting severe AKI greatly improved after combining with uTIM1 and clinical model. After combining with clinical model, the AUCs of sA1AT improved to $0.908(0.846-0.969 ; \mathrm{P}=0.004)$ on performing severe AKI and $0.760(0.694-0.825 ; \mathrm{P}=0.001)$ on performing overall AKI (Table S5, Figure $S 2 A, B$ ). When combining with uTIM1 and clinical model, the AUCs improved to $0.923(0.864-0.981 ; \mathrm{P}=0.019)$ and 0.788 $(0.727-0.850 ; \mathrm{P}=0.001)$, respectively (Table S5, Figure $S 3 A, B)$.

\section{Model performance and VIF analyses}

Finally, we analyzed the model performance and the VIF For the severe AKI model, the estimated HL chi-square was $3.66(\mathrm{P}=0.886)$, and the AUC was 0.908, which has provided 
A

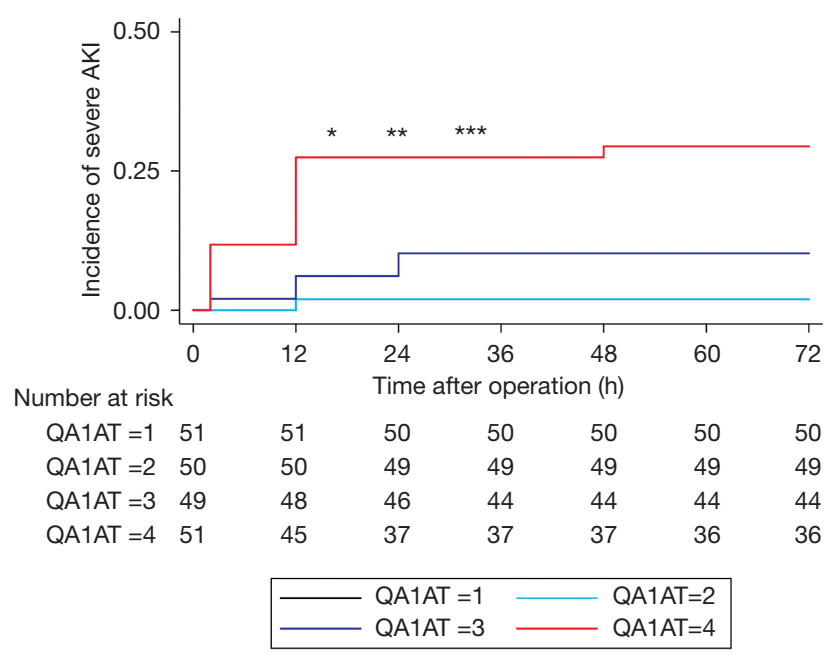

B

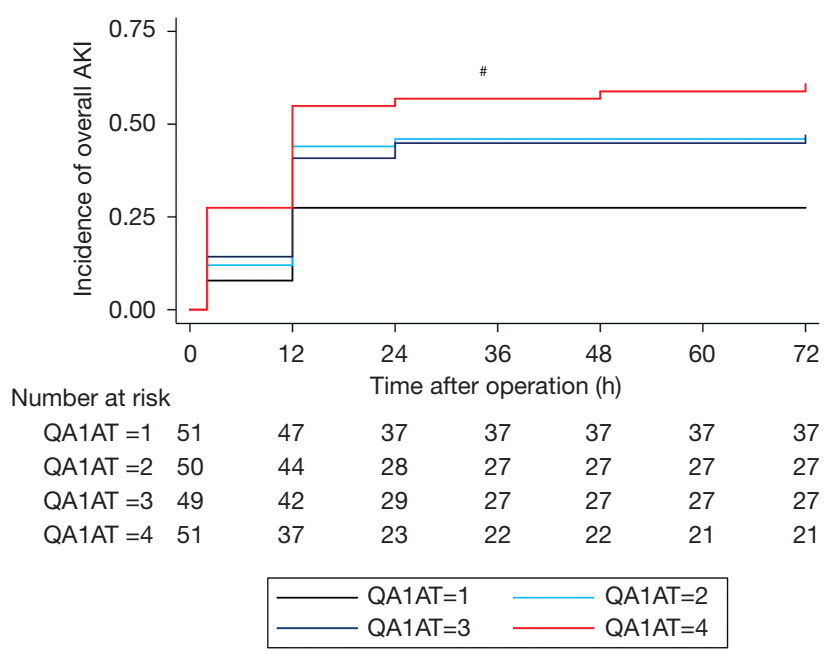

Figure 4 Quartile of sA1AT levels 2 hours after surgery had a graded relationship over time with the incident severe AKI (A) and overall AKI (B) using Kaplan-Meier analyses. (A) The highest quartile of sA1AT had the highest incidence of severe AKI over time. *, P=0.006 versus QA1AT1; **, $\mathrm{P}=0.007$ versus QA1AT2; ***, $\mathrm{P}=0.026$ versus QA1AT3. (B) The highest quartile of sA1AT had the highest incidence of overall AKI over time. *, P=0.003 versus QA1AT1. AKI, acute kidney injury; QA1AT, quartile of alpha1-antitrypsin; sA1AT, serum alpha1antitrypsin.

Table 4 Performance of biomarkers for predicting severe AKI and overall AKI after cardiac surgery ${ }^{a}$

\begin{tabular}{lcc}
\hline \multirow{2}{*}{ Biomarkers } & \multicolumn{2}{c}{ AUC } \\
\cline { 2 - 3 } & AUC $(95 \% \mathrm{Cl})$ & P value $^{\mathrm{b}}$ \\
\hline Predicting severe AKI & $0.814(0.732-0.896)$ & 0.136 \\
sA1AT & $0.712(0.590-0.833)$ & - \\
uTIM1 & \\
Predicting overall AKI & $0.628(0.550-0.705)$ & 0.556 \\
sA1AT & $0.657(0.580-0.734)$ & - \\
uTIM1 &
\end{tabular}

a , biomarkers were measured 2 hours after CPB; ${ }^{\text {, }}$, versus UTIM1. $\mathrm{AKI}$, acute kidney injury; AUC, area under curve; $\mathrm{Cl}$, confidence interval; sA1AT, serum alpha1-antitrypsin; UTIM-1, urinary $\mathrm{T}$ cell immunoglobulin mucin-1.

a good model performance. There was no collinearity among the created variables and the VIFs of the severe AKI model were 1.08-1.52 (Table S6).

\section{Discussion}

In this prospective cohort study, we have demonstrated that
sA1AT measured 2 hours after operation closely associated with severe $\mathrm{AKI}$ and could serves as a wonderful predictor for severe AKI in patients undergoing CPB. Serum A1AT spiked earlier than serum creatinine in patients developed severe AKI after surgery. Higher sA1AT 2 hours after operation tightly associated with an increased risk of severe AKI. Patients with sA1AT (2 hours after CPB) $>2.43 \mu \mathrm{g} / \mathrm{mL}$ presented 6-fold higher hazards of severe AKI compared to the lowest quartile. The AUC of sA1AT for predicting severe AKI was powerful. These data suggest that sA1AT measured immediately after $\mathrm{CPB}$ could provide important early prognostic information.

All the currently existing diagnostic criteria for AKI, including the RIFLE, AKIN, and KDIGO criteria, mainly rely on the serum creatinine level $(2-4,15)$. However, severe injury has already occurred before any increase in the serum creatinine level. Timely diagnosis and early initiation of treatment would improve the clinical course and may yield substantial clinical benefit. A1AT is an acute phase protein, and is involved in the pathophysiologic processes underlying ischemic AKI (13). Until now, few studies have focused on sA1AT values with cardiac surgery-associated AKI. We have delineated the diagnostic value of perioperative sA1AT in this study. The sA1AT nearly reached a peak 2 hours after 
A

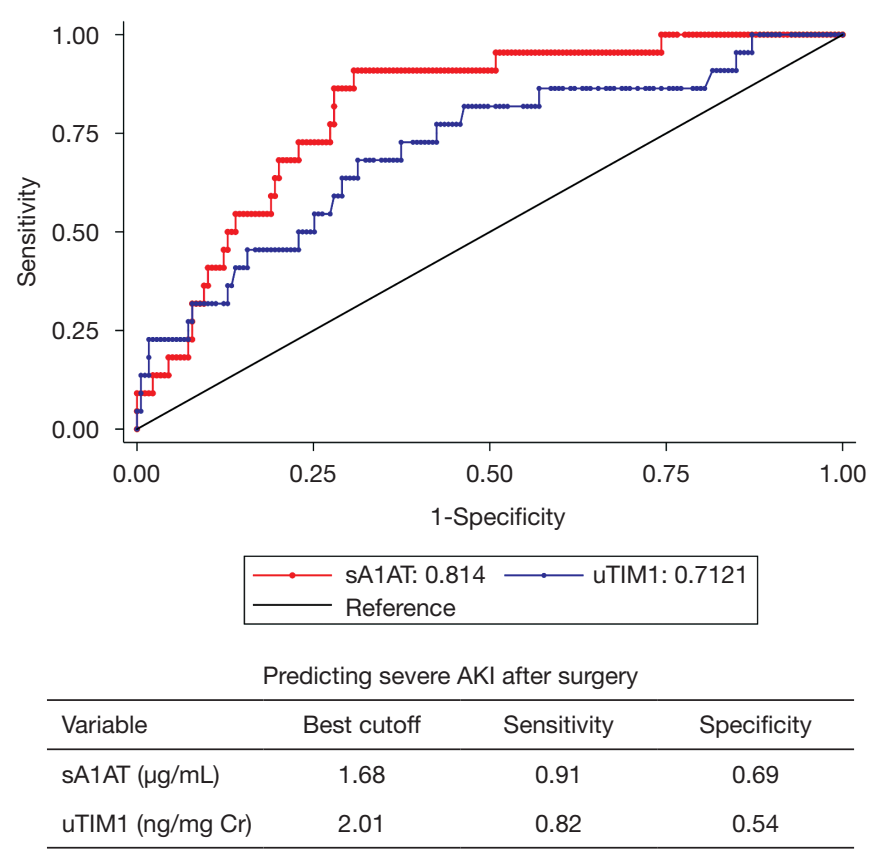

B

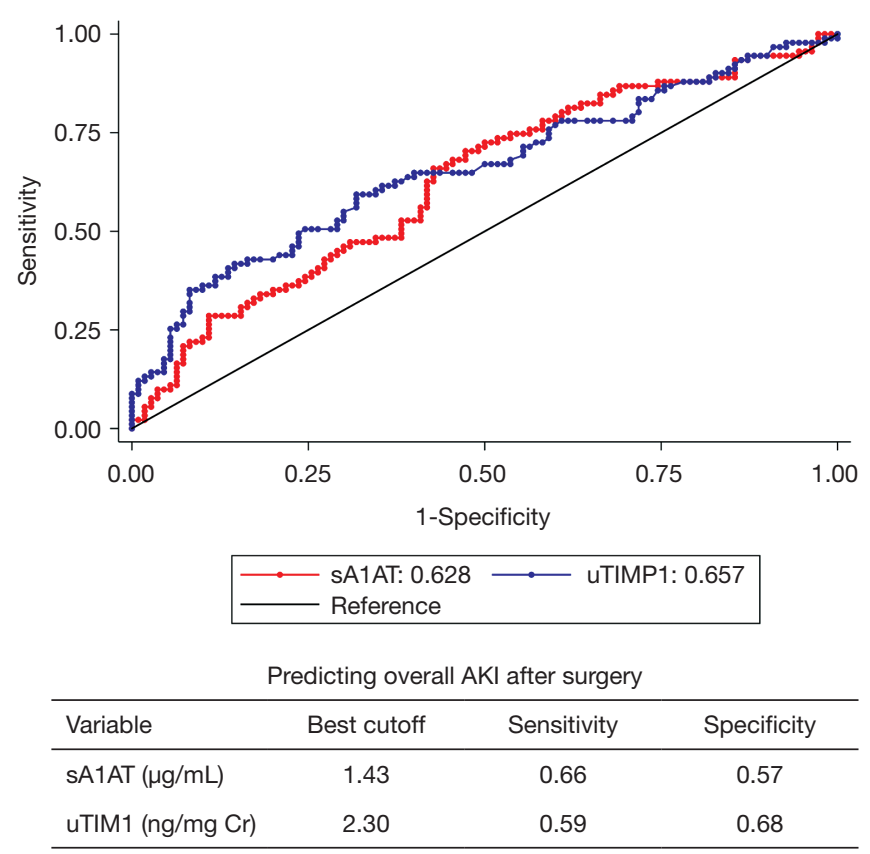

Figure 5 Performance of sA1AT and uTIM1 for predicting severe AKI (A) and overall AKI (B). The best cutoff value, sensitivity value, and specificity value of biomarkers for predicting severe AKI (A) and overall AKI (B) were presented under the curves. AKI, acute kidney injury; sA1AT, serum alpha1-antitrypsin; uTIM1, urinary T cell immunoglobulin mucin-1.

CBP, and remained at this peak for 12 hours in patients who subsequently developed severe AKI, while more than $50 \%$ of the serum creatinine increased 12 hours postoperation. However, uA1AT did not show any significant change in patients with or without AKI. Consistent with the experimental data, we predicted that SA1AT might be an earlier marker for AKI.

During the last decade, several studies have focused on novel biomarkers that could predict AKI after cardiac surgery, such as neutrophil gelatinase-associated lipocalin, uTIM-1, and urinary matrix metalloproteinase-7 (uMMP7) (18-21). However, only a few of the biomarkers have been universally accepted and are currently used in clinical practice. In this study, we noticed that the sA1AT 2 hours after operation closely and independently associated with development of severe AKI. The highest quartile of sA1AT presented the highest severe AKI incidence, which was 16-fold risk than quartile 1 and 2, and 3-fold risk than quartile 3. Collectively, it was evident that the early increased sA1AT was tightly associated with severe AKI after cardiac surgery. It might be an early predictor.

Consistent with previous reports, the performance of these markers for predicting AKI had an AUC of 0.72-0.80 (16-21). In our study, sA1AT 2 hours after operation with an AUC of 0.81 seemed to be a good biomarker for predicting severe AKI, which was better than that of uTIM1. On combining sA1AT with clinical variables, the AUC reached 0.908, which is regarded as pretty performance. Interestingly, the AUC reached 0.923 when we combined the sA1AT with uTIM1 and clinical model. These data supported that sA1AT was a powerful biomarker for predicting severe AKI, and the prognostic value of this marker achieved an exceptionally higher significance after a multi-marker approach was implemented.

Our study had displayed the following strengths and clinical advantages. First, the samples were prospectively collected under standardized conditions and strict criteria. All biomarkers were tested by skilled technicians in a central laboratory to ensure reliability and high quality. Second, sA1AT has been compared with other frequently used biomarkers to ensure the significance of its clinical value. Third, both continuous serum and urine biomarkers were tested simultaneously to determine the changes underlying pathophysiology of A1AT in patients after CPB. The 
quantification of sA1AT is easier (by ELISA kit), which has an advantage of clinical application.

The study also had several limitations. First, urine output was not used for AKI diagnosis because only two patients were anuric and were included in the severe AKI group. Second, the samples were collected and detected at 2, 12, 24 , and 48 hours after operation. The lack of intervening data during other time points (for example, 6 hours after CPB) might be a possible drawback of our study if those data had some significance. Finally, this was a single-center study, and with an unique population hailing from southern China. Further multi-center studies should be considered to verify the value of sA1AT.

\section{Conclusions}

In summary, sA1AT is potentially valuable marker for early prediction of severe AKI development and in-hospital outcomes in patients after cardiac surgery. If further confirmed, measurement of sA1AT could help the clinicians to identify severe post-operative AKI in an earlier stage, initiate appropriate management strategies, and improve clinical outcomes in patients undergoing cardiac surgery.

\section{Acknowledgments}

Funding: This work was supported by the National Natural Science Foundation of China (No. 81770765) and Outstanding Youths Development Scheme of Nanfang Hospital, Southern Medical University (No. 2017J013) to Dr. J Ai, and National Natural Science Foundation of China (No. 81600321) to Dr. S Du.

\section{Footnote}

Conflicts of Interest: The authors have no conflicts of interest to declare.

Ethical Statement: The authors are accountable for all aspects of the work in ensuring that questions related to the accuracy or integrity of any part of the work are appropriately investigated and resolved. This study was approved by the Research Ethics Committee of Nanfang Hospital (No. NFEC-201509-K2), all the participants enrolled in this study provided written informed consent.

\section{References}

1. Huen SC, Parikh CR. Predicting acute kidney injury after cardiac surgery: a systematic review. Ann Thorac Surg 2012;93:337-47.

2. Engoren M, Habib RH, Arslanian-Engoren C, et al. The effect of acute kidney injury and discharge creatinine level on mortality following cardiac surgery. Crit Care Med 2014;42:2069-74.

3. O'Neal JB, Shaw AD, Billings FT. Acute kidney injury following cardiac surgery: current understanding and future directions. Crit Care 2016;20:187.

4. Han SS, Shin N, Baek SH, et al. Effects of acute kidney injury and chronic kidney disease on long-term mortality after coronary artery bypass grafting. Am Heart J 2015;169:419-25.

5. Pickering JW, James MT, Palmer SC. Acute kidney injury and prognosis after cardiopulmonary bypass: a meta-analysis of cohort studies. Am J Kidney Dis 2015;65:283-93.

6. Rosner MH, Okusa MD. Acute kidney injury associated with cardiac surgery. Clin J Am Soc Nephrol 2006;1:19-32.

7. Kumar AB, Suneja M. Cardiopulmonary bypass-associated acute kidney injury. Anesthesiology 2011;114:964-70.

8. Bouma HR, Mungroop HE, de Geus AF, et al. Acute kidney injury classification underestimates long-term mortality after cardiac valve operations. Ann Thorac Surg 2018;106:92-8.

9. Du SL, Zeng XZ, Tian JW, et al. Advanced oxidation protein products in predicting acute kidney injury following cardiac surgery. Biomarkers 2015;20:206-11.

10. Karnaukhova E, Krupnikova SS, Rajabi M, et al. Heme binding to human alpha-1 proteinase inhibitor. Biochim Biophys Acta 2012;1820:2020-9.

11. Zager RA, Johnson AC, Frostad KB. Combined iron sucrose and protoporphyrin treatment protects against ischemic and toxin-mediated acute renal failure. Kidney Int 2016;90:67-76.

12. Jonigk D, Al-Omari M, Maegel L, et al. Antiinflammatory and immunomodulatory properties of a1antitrypsin without inhibition of elastase. Proc Natl Acad Sci 2013;110:15007-12.

13. Janciauskiene S, Tumpara S, Wiese M, et al. Alpha1antitrypsin binds hemin and prevents oxidative activation of human neutrophils: putative pathophysiological significance. J Leukoc Biol 2017;102:1127-41.

14. Janciauskiene $S$, Wrenger $S$, Immenschuh $S$, et al. The Multifaceted Effects of Alpha1-Antitrypsin on Neutrophil Functions. Front Pharmacol 2018;9:341.

15. Kellum JA, Lameire N, Aspelin P, et al. KDIGO Clinical Practice Guideline for Acute Kidney Injury 2012. Kidney 
Int Suppl 2012;2:1-138.

16. Hazle MA, Gajarski RJ, Aiyagari R, et al. Urinary biomarkers and renal near-infrared spectroscopy predict intensive care unit outcomes after cardiac surgery in infants younger than 6 months of age. J Thorac Cardiovasc Surg 2013;146:861-867.e1.

17. Elmedany SM, Naga SS, Elsharkawy R, et al. Novel urinary biomarkers and the early detection of acute kidney injury after open cardiac surgeries. J Crit Care 2017;40:171-7.

18. Kaufeld T, Foerster KA, Schilling T, et al. Preoperative serum uric acid predicts incident acute kidney injury following cardiac surgery. BMC Nephrol 2018;19:161.

Cite this article as: Du S, Tian J, Xiao Z, Luo Z, Lin T, Zheng S, Ai J. Serum alpha 1-antitrypsin predicts severe acute kidney injury after cardiac surgery. J Thorac Dis 2019;11(12):50535062. doi: 10.21037/jtd.2019.12.17
19. Zelt JGE, Mielniczuk LM, Liu PP, et al. Utility of Novel Cardiorenal Biomarkers in the Prediction and Early Detection of Congestive Kidney Injury Following Cardiac Surgery. J Clin Med 2018;7:540.

20. Krawczeski CD, Goldstein SL, Woo JG, et al. Temporal relationship and predictive value of urinary acute kidney injury biomarkers after pediatric cardiopulmonary bypass. J Am Coll Cardiol 2011;58:2301-9.

21. Yang X, Chen C, Teng S, et al. Urinary Matrix Metalloproteinase-7 Predicts Severe AKI and Poor Outcomes after Cardiac Surgery. J Am Soc Nephrol 2017;28:3373-82. 


\section{Supplementary}

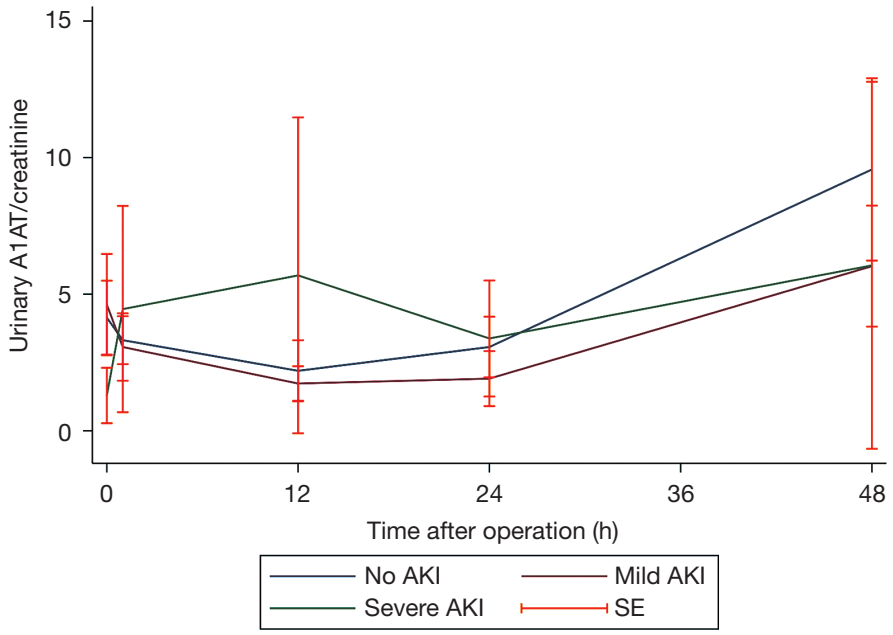

Figure S1 Changes of urine A1AT levels after surgery. Urine A1AT level did not change over time after cardiac surgery in patients with or without AKI. Data are shown as mean \pm SD. AKI, acute kidney injury; SD, standard difference; uA1AT, urine alpha 1-antitrypsin.

Table S1 Multivariate logistic regression analyses of sA1AT for predicting overall AKI after cardiac surgery ${ }^{2}$

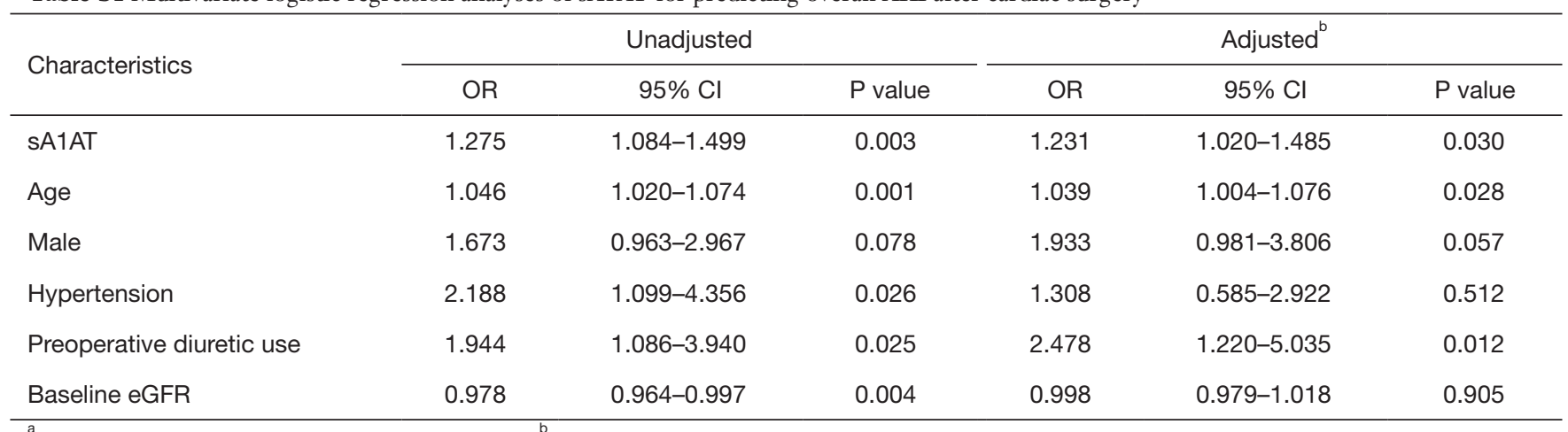

a , serum A1AT expressed 2 hours after CPB; ${ }^{b}$, adjusted for age, male, history of hypertension, preoperative diuretic use, preoperative eGFR, and CPB time. AKI, acute kidney injury; CPB, cardiopulmonary bypass; eGFR, evaluated glomerular filtrated rate; sA1AT, serum alpha 1-antitrypsin. 
Table S2 Multivariate cox regression analyses of categorized sA1AT (Versus Q2 or Q3) for predicting severe AKI after cardiac surgery ${ }^{2}$

\begin{tabular}{|c|c|c|c|c|}
\hline sA1AT & \multicolumn{4}{|c|}{ Severe AKI } \\
\hline \multicolumn{5}{|l|}{ Versus Q2 } \\
\hline 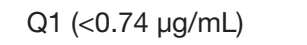 & $0.98(0.06-15.67)$ & 0.989 & $1.25(0.08-20.47)$ & 0.875 \\
\hline Q2 (0.74-1.21 $\mu \mathrm{g} / \mathrm{mL})$ & 1.0 (reference) & - & 1.0 (reference) & - \\
\hline Q4 (>2.43 $\mu \mathrm{g} / \mathrm{mL})$ & $16.43(2.17-124.48)$ & 0.007 & $7.59(0.96-59.94)$ & 0.055 \\
\hline \multicolumn{5}{|l|}{ Versus Q3 } \\
\hline 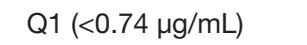 & $0.19(0.02-1.61)$ & 0.127 & $1.00(0.95-1.04)$ & 0.632 \\
\hline Q2 (0.74-1.21 $\mathrm{gg} / \mathrm{mL})$ & $0.18(0.02-1.64)$ & 0.132 & $1.12(0.42-2.97)$ & 0.492 \\
\hline
\end{tabular}

a , sA1AT expressed 2 hours after operation; ', adjusted for age, sex, BMI, SBP, preoperative eGFR, and CPB time. AKI, acute kidney injury; $\mathrm{BMI}$, body mass index; $\mathrm{Cl}$, confidence interval; $\mathrm{CPB}$, cardiopulmonary bypass; eGFR, evaluated glomerular filtrated rate; HR, hazards ratio; $\mathrm{Q}$, quartile; SA1AT, serum alpha 1-antitrypsin; SBP, systolic blood pressure.

Table S3 Multivariate cox regression analyses of sA1AT for predicting overall AKI after cardiac surgery

\begin{tabular}{|c|c|c|c|c|c|}
\hline sA1AT & AKI (\%) & \multicolumn{4}{|c|}{ Primary outcome: overall AKI } \\
\hline Q1 (<0.74 $\mu \mathrm{g} / \mathrm{mL})$ & 27.5 & 1.0 (reference) & - & 1.0 (reference) & - \\
\hline 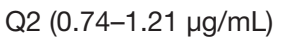 & 46.0 & $1.76(0.91-3.43)$ & 0.094 & $1.52(0.77-2.99)$ & 0.224 \\
\hline 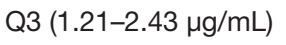 & 46.9 & $1.82(0.94-3.54)$ & 0.077 & $1.30(0.65-2.63)$ & 0.457 \\
\hline$P$ for trend & 0.002 & & & & \\
\hline
\end{tabular}

Table S4 Multivariate logistic regression analyses of sA1AT for predicting length in ICU stay and in hospital stay after cardiac surgery ${ }^{2}$

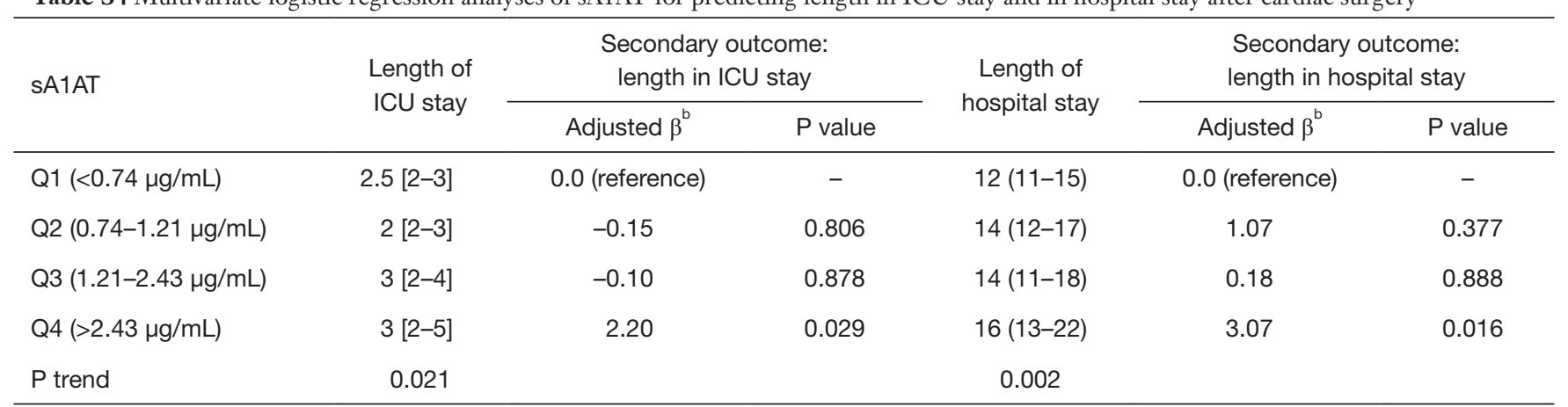

a , sA1AT expressed 2 hours after operation; ${ }^{\text {, }}$, adjusted for age, sex, BMI, SBP, preoperative eGFR, and CPB time. BMI, body mass index; CPB, cardiopulmonary bypass; eGFR, evaluated glomerular filtrated rate; ICU, intensive care unit; Q, quartile; sA1AT, serum alpha1antitrypsin; SBP, systolic blood pressure. 
Table S5 Performance of combination of clinical model for predicting severe AKI and overall AKI after cardiac surgery ${ }^{\mathrm{a}}$

\begin{tabular}{|c|c|c|c|}
\hline Biomarkers & \multicolumn{3}{|c|}{ AUC (95\% Cl) } \\
\hline \multicolumn{4}{|c|}{ Predicting severe AKI } \\
\hline sA1AT & $0.814(0.732-0.896)$ & $0.908(0.846-0.969)$ & 0.004 \\
\hline uTIM1 & $0.712(0.590-0.833)$ & $0.897(0.812-0.981)$ & 0.011 \\
\hline \multicolumn{4}{|c|}{ Predicting overall AKI } \\
\hline sA1AT & $0.628(0.550-0.705)$ & $0.760(0.694-0.825)$ & 0.001 \\
\hline uTIM1 & $0.657(0.580-0.734)$ & $0.777(0.714-0.840)$ & 0.002 \\
\hline sA1AT + uTIM1 & $0.675(0.599-0.751)$ & $0.788(0.727-0.850)$ & 0.001 \\
\hline
\end{tabular}

, biomarkers were measured 2 hours after CPB; ${ }^{b}$, clinical model was comprised of age, sex, BMI, preoperative SBP, preoperative eGFR, and CPB time; ', biomarkers plus clinical model versus clinical model alone. AKI, acute kidney injury; AUC, area under curve; BMI, body mass index; CPB, cardiopulmonary bypass; eGFR, evaluated glomerular filtrated rate; SA1AT, serum alpha1-antitrypsin; SBP, systolic blood pressure; UTIM-1, urinary T cell immunoglobulin mucin-1.

A

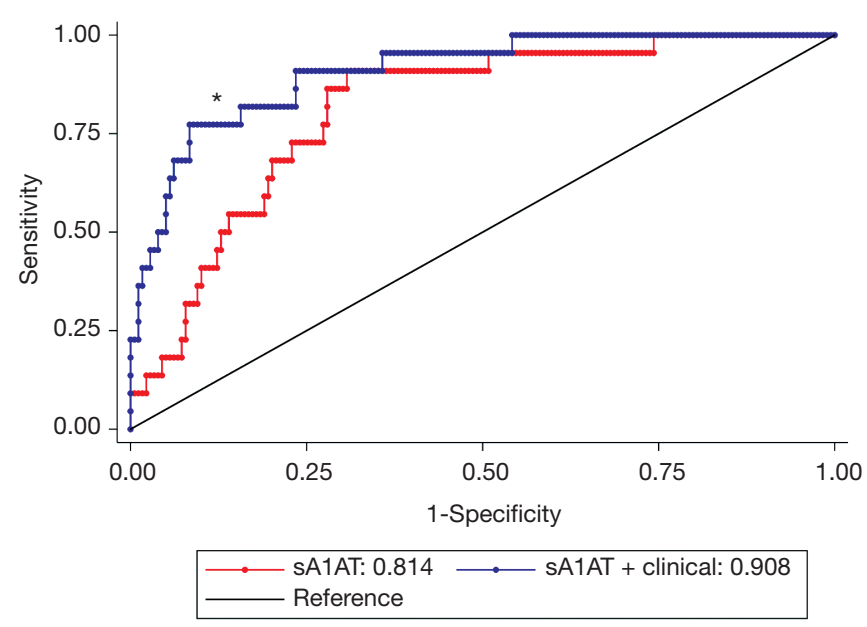

B

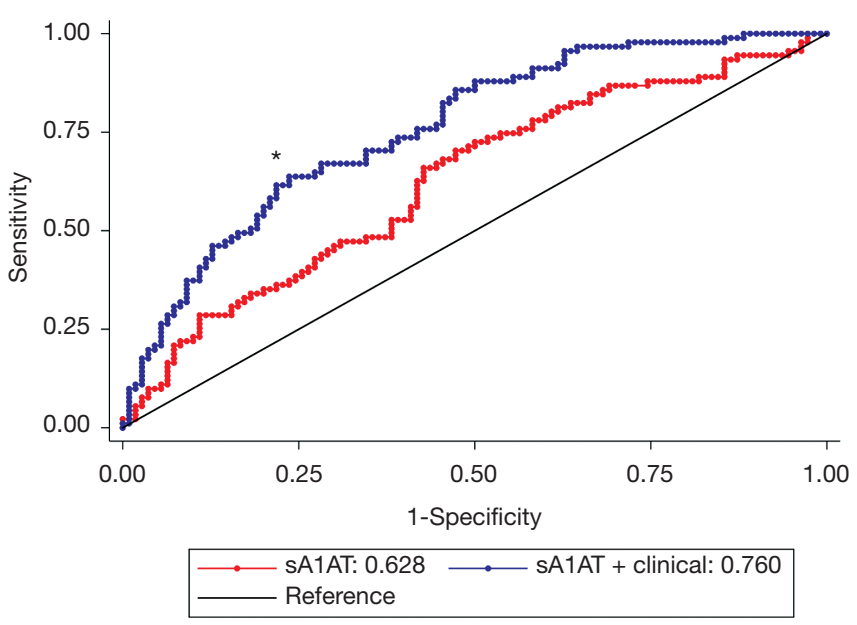

Figure S2 Performance of combination of sA1AT and clinical model for predicting severe AKI (A) and overall AKI (B) after cardiac surgery. Clinical model was comprised of age, sex, body mass index, systolic blood pressure, preoperative eGFR, and cardiopulmonary bypass time. *, $\mathrm{P}<0.01$ versus sA1AT alone in A and $\mathrm{B}$. AKI, acute kidney injury; eGFR, evaluate glomerular filtrated rate; sA1AT, serum alpha 1-antitrypsin. 


\section{A}

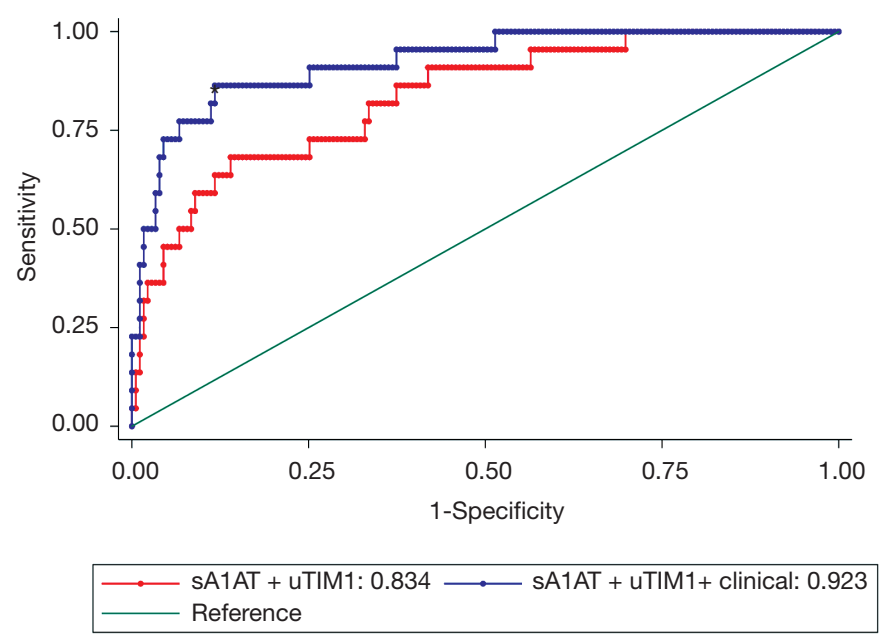

B

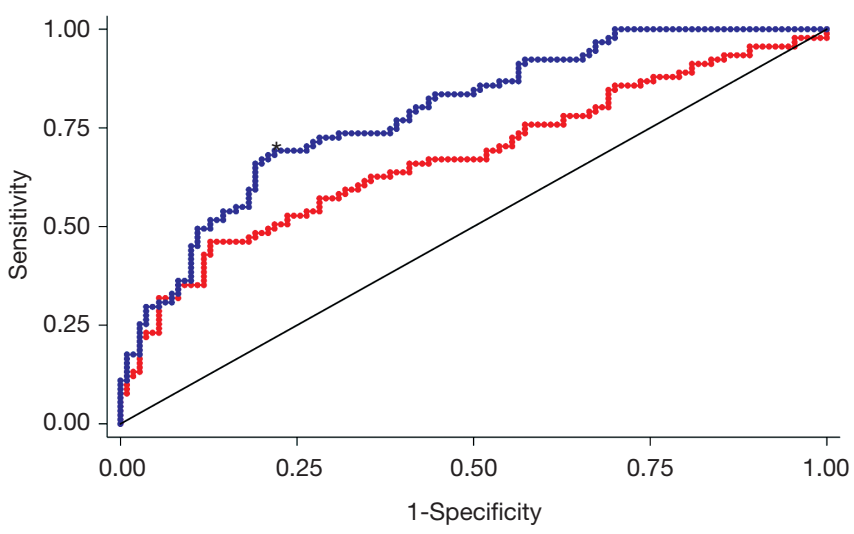

sA1AT + UTIM1: 0.675 Reference

Figure S3 Performance of combination of sA1AT, uTIM1, and clinical model for predicting severe AKI (A) and overall AKI (B) after cardiac surgery. Clinical model was comprised of age, sex, body mass index, systolic blood pressure, preoperative eGFR, and cardiopulmonary bypass time. *, $\mathrm{P}<0.05$ versus sA1AT + uTIM1 in A and B. AKI, acute kidney injury; eGFR, evaluate glomerular filtrated rate; sA1AT, serum alpha 1-antitrypsin; uTIM1, urinary T cell immunoglobulin mucin-1.

Table S6 Evaluated VIF for multicollinearity analysis among created variables

\begin{tabular}{lc}
\hline Variables & VIF \\
\hline eGFR & 1.52 \\
Age & 1.49 \\
CPB time & 1.22 \\
SBP & 1.11 \\
Sex & 1.09 \\
BMI & 1.08 \\
SA1AT & 1.11 \\
\hline
\end{tabular}

BMI, body mass index; CPB, cardiopulmonary bypass; eGFR, evaluated glomerular filtrated rate; SA1AT, serum alpha 1-antitrypsin; SBP, systolic blood pressure; VIF, variance inflation factor. 